\title{
Application of multiplexed pharmacokinetic immunoassay to quantify in vivo drug forms and coadministered biologics
}

\author{
Nicole Woodbury*,1, Eric Bald ${ }^{1}$, Brian Geist ${ }^{1} \&$ Tong-Yuan Yang ${ }^{1}$ \\ ${ }^{1}$ Biologics Development Sciences, Janssen BioTherapeutics, Janssen R\&D LLC, Spring House, PA 19477, USA \\ *Author for correspondence: nwoodbur@its.jnj.com
}

\begin{abstract}
Aim: Meso Scale Discovery U-PLEX ${ }^{\circledR}$ provides an opportunity to develop multiplexed pharmacokinetic (PK) immunoassays. Two case studies demonstrate the utility of multiplexed PK methods. Materials \& methods: Development of PK ligand-binding assays quantify of nonclinical plasma concentrations of a biotherapeutic that has degraded due to in vivo biotransformation, and clinical serum concentrations from two biotherapeutics spiked into a single sample. Results: Data from multiplexed U-PLEX PK methods are comparable to results from single-readout streptavidin Meso Scale Discovery gold PK methods. Multiplex measurement of a nonclinical study showed acceptable performance for accuracy, precision and dilutional linearity while a clinical study additionally passed selectivity, specificity and stability. Conclusion: Regulated, validation-ready multiplex PK methods for both nonclinical and clinical studies allow opportunities for high-throughput bioanalysis.
\end{abstract}

First draft submitted: 18 June 2019; Accepted for publication: 21 October 2019; Published online: 17 December 2019

Keywords: bioanalysis $\bullet$ immunoassay $\bullet$ ligand binding assay $\bullet$ multiplex $\bullet$ pharmacokinetics $\bullet$ validation

Multiplexing bioanalysis, a single technical process with the ability to generate multiple quantitative measurements from a single sample, is a widely applied bioanalytical concept during the discovery and development of biopharmaceuticals [1]. Multiplexing bioanalysis offers gains not only in technical efficiency through reduction in time and resources associated with bioanalysis but also provides a solution for volume-limited samples. LC-MS/MS methodology has leveraged multiplexing primarily through differentiation of analytes based on their physicochemical properties [2], while quantitative PCR uses highly specific and selective primers and probes targeting the genes of interests [3]. In addition, Luminex ${ }^{\circledR}$ bead-based multiplexing assay has shown to be successful with biomarker detection [4]. To achieve the desired assay selectivity and specificity in multiplexing testing, immunoassay methods typically use analyte-specific detection reagents labeled with biochemical tags that produce a distinct signal dependent on method format and instrument platform [5]. Acceptance criteria for typical assay parameters outlined by regulatory guidelines provide the basis for method validation of ligand-binding assays (LBAs), which will be implemented in regulated bioanalysis of biologics concentration measurement. Most recently, US FDA guidance for bioanalytical method validation outlines the general guidelines for the experimentally determined accuracy, precision, selectivity, specificity and robustness of a bioanalytical method, which are required to support any pivotal GLP and clinical studies used to demonstrate the safety and effectiveness of a drug [6].

The number of biologics (protein therapeutics) in clinical development has exponentially increased over the last 20 years due to its favorable probability of commercial launch [7]. More complex molecular modalities, such as multispecific and protein fusion biologics, are being engineered in development to target multiple disease mechanisms to improve product efficacy and the likelihood for therapeutic success. Contrary to multispecific biologics, the trend toward combination therapies (coadministration of two biologics targeting a similar biological mechanism or complementing pathways in a known disease pathology) has garnered greater interest. This approach allows for independent titration of multitarget disease therapy that may achieve a more meaningful clinical outcome through an additive or synergistic pharmacological effect in vivo. To date, little has been published on the use of multiplexing pharmacokinetic (PK) methods for biologics. However, these approaches can potentially provide 
several advantages given that the complexity of current biologic molecules may require multiple bioanalytical readouts to fully understand the PK profile from 'first in human' through Phase III clinical studies. Developing multiplexing LBA methods, however, comes with both common and unique bioanalytical challenges. The potential for matrix interference or lack of reagent specificity could be among the challenges seen when developing a multiplex PK method, similar to what is observed in fluorescence-tagged immunoassays commonly used in biomarker detection [2].

Multiplexing approaches have been primarily used for the immunoassay detection of protein biomarkers [5]. The multiplexing format is achieved with detection reagents in a mixed solution, specifically conjugated to different fluorescence tags, binding to its protein analyte. A common challenge in this type of multiplexed immunoassay is interference caused by the emission from different fluorescence tags resulting in potentially inaccurate measurements of intended analyte [2]. Meso Scale Discovery (MSD) U-PLEX ${ }^{\circledR}$ technology provides an alternative approach for developing multiplex immunoassays by eliminating different fluorescence tags. This platform uses ten microspots per well on a 96-well plate to physically separate out each protein analyte with proprietary-coating technology of one specific capture reagent per microspot $[8]$.

Herein, we demonstrate the capability to develop multiplex PK methods with the U-PLEX technology for the quantitative bioanalysis of biotherapeutics that could have a significant beneficial impact during different stages of the drug development process [9]. In case study 1 , a therapeutic peptide-monoclonal antibody (mAb) fusion molecule (a functional peptide moiety chemically linked to a fully human antibody framework for halflife extension) was being investigated as a potential therapeutic during the lead candidate selection stage. A critical question was to understand the kinetics of in vivo biotransformation and stability of the lead candidate by providing PK measurement of 'active' drug versus 'total' drug. The in vivo biotransformation can cause a $N$-terminus degradation to the peptide portion of this fusion molecule, leading to the lack of binding to the intended target. Since these in vivo stability assessments were conducted in either small animal or nonhuman primate studies, the use of multiplexing PK assays for 'active' and 'total' drug measurement simultaneously in the same sample provides an advantage over using two single assay readouts due to limited study sample volume required for the bioanalysis. For case study 2, the ability to incorporate a multiplexing PK approach provides improved efficiency during bioanalysis when two different biotherapeutics are being investigated as a combination therapy in clinical studies. For the purposes of case study 2, we used two mAbs binding to different cell surface targets often found overexpressed in a common disease population. Combination therapies of two or more biologics have become increasingly prevalent due to the potential for improved therapeutic response attributed to each biologic dosed in the study and can yield greater clinical outcomes through additive or synergistic effects, targeting two or more disease-associated pathways [10-15]. Coadministration of two biologics has proven effective both in vitro and in vivo during nonclinical and clinical studies [14]. During clinical phase studies, performing bioanalysis on study samples collected during a coadministration study requires the development and validation of two individual clinical PK assay methods to measure each compound separately. Multiplexing PK methods, illustrated in Figure 1, would enable the simultaneous quantitation of drug concentrations from both biologics in the same sample. In addition, we have compared the performance of the U-PLEX multiplex PK assay with the standard single-readout MSD PK methods using standard gold plates to provide confidence that intra- and interstudy results are comparable when changing assay technologies.

\section{Materials \& methods}

\section{Regression \& acceptance criteria}

All experiments were regressed using Fifth Party Logistics (5PL) with $1 / \mathrm{Y}^{2}$ weighting in Watson LIMS. Acceptance criteria for accuracy and precision are defined by mean percent bias (\%bias), percent of coefficient of variation $(\% \mathrm{CV})$ and total error (TE). Since research-based pre-new molecular entity (NME) testing, as in case study 1 , is exploratory, has a rapid turnover and results not used for health authority submission purposes, the adapted in-house research assay acceptance criteria are wider than the regulated authorities require and have been adapted as followed: \%bias is $\pm 25 \%$ for upper limit of quantification (ULOQ), high quality control (HQC), mid quality control (MQC), and low quality control (LQC) meanwhile $\pm 30 \%$ for lower limit of quantification (LLOQ). HQC, MQC and LQC are confirmed in duplicate on three independent plates. ULOQ and LLOQ need to be confirmed only once. Selectivity testing is not required for research method assessments. Dilutional linearity (DL) must have two dilutions fall within the range of quantification and $\pm 25 \%$ bias of nominal concentration. For all data points, acceptance criteria for CV and TE is 25 and 35\%, respectively. 


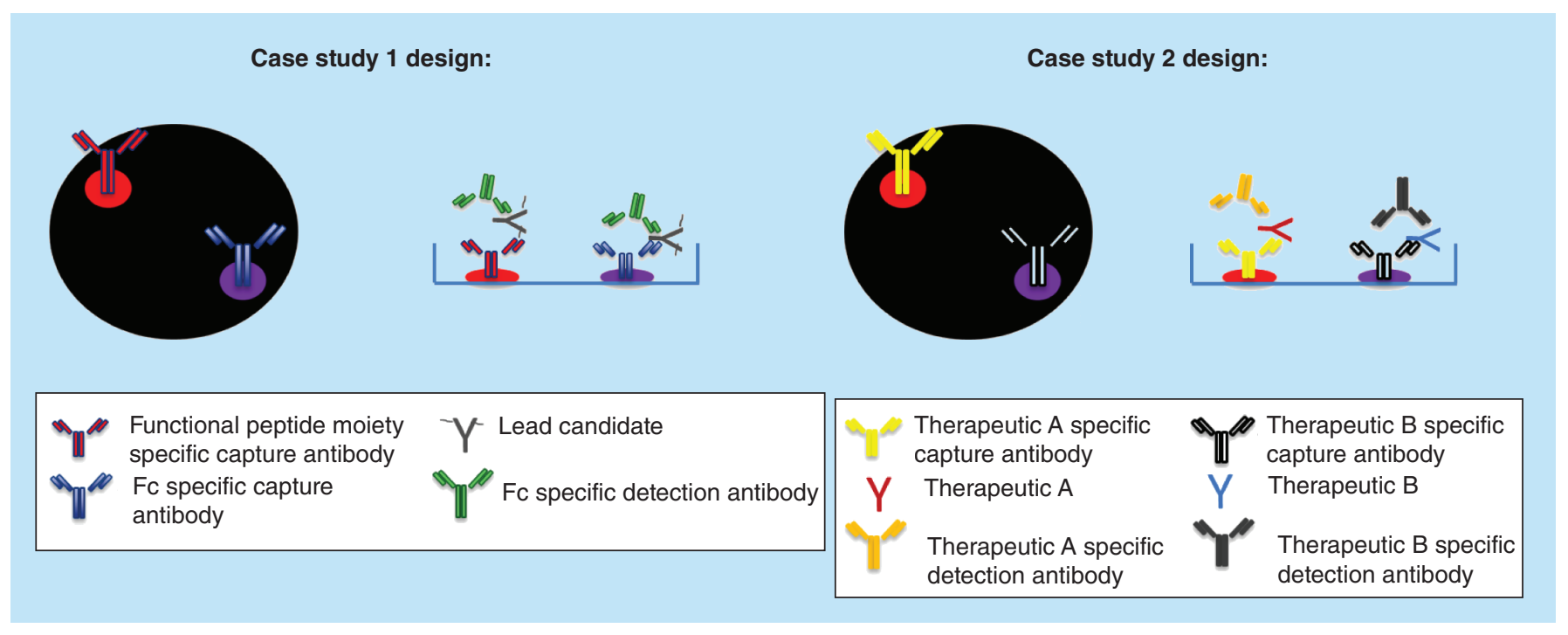

Figure 1. Study designs for both case studies 1 and 2 for illustration purposes.

Post-NME nonclinical and clinical assays, such as case study 2, acceptance criteria are defined as follows: \%bias is $\pm 20 \%$ for ULOQ, HQC, MQC and LQC meanwhile $\pm 25 \%$ for LLOQ. All quality control (QC) levels are confirmed in duplicate on six independent plates. Selectivity testing was completed in ten individuals, splits into five males and five females. For clinical testing, disease patient individuals were also included in selectivity testing along with healthy patients. DL must have two dilutions fall within the range of quantification and $\pm 20 \%$ bias of nominal concentration. For all data points, acceptance criteria for $\mathrm{CV}$ and $\mathrm{TE}$ are 20 and 35\%, respectively.

\section{Reagent \& chemicals}

U-PLEX Development Pack containing plates, linkers, stop solution and read buffer (Catalog: K15235N) were purchased from MSD as well as sulfo-tag (Catalog: R91AO). Low cross buffer (Catalog: 100500), utilized as assay buffer throughout the procedure, was obtained from Candor. Pooled human serum (Catalog: HUMANSRM) as well as healthy and diseased individual serum (Catalogs: HMBIOSRM-M; HMBIOSRM-F) were bought from BioIVT Inc. Purchased from BioIVT was also the pooled cynomolgus monkey (cyno)plasma (K2EDTA) (NHP01PLK2PNN) used in the lead candidate selection feasibility. EZ-Link biotin (Catalog: 21335) was bought from Thermo Fisher Scientific. Parent antibodies used for capture and detection were generated in-house.

\section{QCs \& standard calibrators}

For case study 1, lead candidate degradation characterization standard calibrators and QCs prepared as follows: Therapeutic $Y$ was diluted in cynoplasma at $1.25 \mu \mathrm{g} / \mathrm{ml}$ and twofold serially diluted in $100 \%$ cynoplasma to create an additional nine concentrations that formed the calibrators of a standard curve. A blank control of cynoplasma only was included in the assay for signal background monitoring. QCs were prepared in cynoplasma with the following concentrations: $1250,93,750,11,718.75,2929.69$ and $9.77 \mathrm{ng} / \mathrm{ml}$.

For case study 2, standard calibrators and QCs were prepared as follows: Therapeutic A was diluted in low cross buffer at $6.93 \mu \mathrm{g} / \mathrm{ml}$ and threefold serially diluted to create an additional nine concentrations that formed the calibrators of a standard curve. Therapeutic B was diluted in low cross buffer with $5 \%$ human serum at $0.512 \mu \mathrm{g} / \mathrm{ml}$ and twofold serially diluted to create an additional nine concentrations that formed the calibrators of a standard curve. A nonspiked standard calibrator was incorporated into each respective standard calibrator, Low cross buffer only for Therapeutic A and 5\% human serum only for Therapeutic B, for background monitoring. QC calibrators containing both therapeutics were generated by the following: Therapeutic A 80-fold QCs had the following concentrations: 291.6, 220, 20,1.2, $0.4 \mu \mathrm{g} / \mathrm{ml}$. Therapeutic B 80-fold QCs had the following concentrations: $20.48,16,2.56,0.96,0.32 \mu \mathrm{g} / \mathrm{ml}$. QCs for both therapeutics were created at 80 -fold nominal concentration, so equal parts of each therapeutic could be mixed together. After this mix occurs, the minimum required dilution (MRD) is 40 for all QCs for both therapeutics. 


\section{Selectivity \& DL reagent \& chemicals}

Therapeutic A's LLOQ is $0.005 \mu \mathrm{g} / \mathrm{ml}$. Therapeutic B's LLOQ is $0.004 \mu \mathrm{g} / \mathrm{ml}$. The MRD of the assay is 40 , meaning these samples need to be concentrated $40 \times$ their LLOQ. In neat serum, concentrations of Therapeutic $\mathrm{A}$ and $\mathrm{B}$ are 0.20 and $0.16 \mu \mathrm{g} / \mathrm{ml}$, respectively. Ten naive healthy and ten diseased individuals were prepared containing both Therapeutic A and Therapeutic B.

The DL sample for case study 2 was prepared with $2070 \mu \mathrm{g} / \mathrm{ml}$ of Therapeutic A and $5 \mu \mathrm{g} / \mathrm{ml}$ of Therapeutic B in pooled human serum. The DL sample for case study 1 was prepared at $11.76 \mu \mathrm{g} / \mathrm{ml}$ of Therapeutic $\mathrm{Y}$ in pooled cynoplasma.

\section{Sample dilution}

For case study 1, manual dilution of all QC levels underwent a 1/5 dilution in low cross buffer. Subsequent 1/100 dilution, final dilution of $1 / 500$, for HQC, MQC and LQC were completed in $20 \%$ cynoplasma. Cynoplasma was diluted to $20 \%$ in low cross buffer. The DL sample would undergo a $1 / 5,1 / 100$ and $1 / 5$ dilution scheme resulting in a final dilution factor of 2500 . The second and third dilution would also be completed in the $20 \%$ cynoplasma.

For case study 2, an automated liquid handler added equal parts of each therapeutic's standard calibrator into a 1-ml deepwell and QC of each therapeutic into $14 \mathrm{ml}$ snap cap tubes. QCs were diluted at the MRD (1/40 dilution) allowing the QCs to fall within the quantifiable range. Selectivity was tested at just the MRD $(1 / 40$ dilution). DL sample had three $1 / 40$ sample dilutions completed. This dilution scheme allowed for two of the dilutions for each therapeutic to fall within the range of quantification.

\section{Biotin \& SulfoTag reagents}

Therapeutic A, B and Y each had in-house generated anti-idiotypic antibodies (anti-ids) identified for use. Each of the anti-ids used were previously screened using Octet (data not shown) for specificity of the reagent to their respective and intended target.

\section{U-PLEX-coupled antibody solution}

Creation of U-PLEX-coupled antibody solution followed manufacturer's quick guide preparation recommendations [8]. Coupled antibody solution refers to the solution that binds your biotin-labeled antibody to the proprietary MSD linker.

Therapeutic A and Therapeutic B's biotinylated anti-ids were prepared separately and bound to two unique linkers. Respectively, the 'active' and 'total' biotinylated anti-ids were also linked to two unique linkers. The following process was used to create linker solution for each capture antibody:

Each biotinylated capture anti-id was prepared in $200 \mu \mathrm{l}$ of low cross buffer to yield a final concentration of $10 \mu \mathrm{g} / \mathrm{ml}$. Upon completion, $300 \mu \mathrm{l}$ of the assigned unique linker was added, vortexed and incubated for $30 \mathrm{~min}$ with shaking at $600 \mathrm{rpm}$ on orbital shaker at room temperature. At completion of incubation, $200 \mu \mathrm{l}$ of stop solution was added, vortexed and incubated for $30 \mathrm{~min}$ with shaking at $600 \mathrm{rpm}$ on orbital plate shaker at room temperature to complete the reaction.

For each experiment in case study 1, linker preparation was independent and completed same day prior to beginning plate procedure. Case study 2 had a single experiment to test the manufacture's stability claim that linkers can be stored up to 7 days at $2-8^{\circ} \mathrm{C}$. Besides this stability experiment, linkers were prepared independently and on same day as experiment completion.

\section{Master mix creation}

For U-PLEX plates, the following steps were used for a single plate's minimum volume required of master mix: Master mix, defined as solution with both capture and detection reagent, was created in low cross buffer for both case studies. $600 \mu \mathrm{l}$ of coupled antibody solutions for each linker was added to $4.8 \mathrm{ml}$ of low cross buffer yielding a total volume of $6 \mathrm{ml}$. SulfoTag reagent(s) were added, so final working concentration was $1 \mu \mathrm{g} / \mathrm{ml}$ in both case studies. Master mix was vortexed and protected from light until use. These reagents were scaled up maintaining the ratios of coupled antibody solution to low cross buffer and a final working concentration of $1 \mu \mathrm{g} / \mathrm{ml}$ SulfoTag-labeled reagent(s) when more than one assay plate was run.

MSD gold plates used the following calculations for a single plates minimum volume: Biotinylated anti-ids as well as SulfoTag anti-ids were added, so final working concentration was $1 \mu \mathrm{g} / \mathrm{ml}$ in low cross buffer. Master mix 
was vortexed and protected from light until ready to be plated. These volumes were scaled up if more than one assay plate was to be ran.

\section{MSD gold \& U-PLEX assay procedure}

Both case studies followed a homogenous plate-based procedure. The assay plate was blocked with low cross buffer and allowed to incubate at room temperature, sealed, for $30 \mathrm{~min}$ while shaking at $650 \mathrm{rpm}$ on an orbital shaker. After blocking incubation, the assay plate was inverted to empty all contents and tapped dry on a clean absorbent pad.

For both case studies, samples at their final dilutions previously described in the 'Sample dilution' section were then added in duplicate to the plate. $10 \mu \mathrm{l}$ of calibrators/QCs/samples (such as DL or selectivity) and $40 \mu \mathrm{l}$ master mix, previously described in the 'Master mix creation' section, were added to plate. Assay plate was sealed, protected from light and incubated for $1 \mathrm{~h}$ at room temperature with $650 \mathrm{rpm}$ shaking. Assay plate was then washed using an automatic plate washer with $1 \times$ PBS $+0.05 \%$ Tween-20 (Janssen R\&D) solution.

$150 \mu \mathrm{l}$ per well of $1 \times$ MSD Read buffer was added to the assay plate. The assay plate was then read on an MSD S 600 Imager.

\section{Results}

\section{Case study 1}

Multiplex PK method for lead candidate therapeutic $Y$ characterization

To quantitate the 'active' and 'total' of Therapeutic $\mathrm{Y}$ and its associated in vivo degradation, the multiplex assay format incorporated two different capture antibodies targeting distinct domains of the peptide-mAb construct. The measurement of 'active' drug is determined by a method developed to quantify the biologically active portion of the therapeutic by capturing the functional portion of the peptide associated with activity while the measurement of 'total' drug is defined by a method developed to quantify total Fc framework within the sample. The first capture antibody is specific to an epitope associated with the active domain of the peptide moiety intended to measure 'active' drug (e.g., intact form with two active peptides and the drug form with only one single active peptide due to in vivo degradation of second peptide), while the second capture antibody is specific to the framework of the Fc domain intended to measure 'total' drugs (e.g., intact drug, drug form with single peptide and drug form without any peptide). Both antibodies are conjugated to different linkers that will position them on different microspots in the same well of a U-PLEX 96-well plate. After the capture step, a common antihuman Fc detection antibody conjugated with ruthenium is used to detect the 'capture antibody-drug (degraded or not)' complexes. The electrochemiluminescence (ECL) signal generated from each microspot is acquired by MSD plate reader separately and is proportional to the concentrations of drug types (active or total) in the sample. The accuracy and precision of 'active' and 'total' drug molecule quantification was assessed in cynomolgus monkey plasma.

\section{Accuracy \& precision}

Accuracy and precision are critical assay parameters to determine the reliability and reproducibility of a given bioanalytical method.

Evaluation of immunoassay performance to measure 'active' and 'total' drug on both standard streptavidin MSD gold and U-PLEX plates was conducted, and accuracy and precision was assessed between the two plate types. Five concentration levels of QCs (ULOQ, HQC, MQC, LQC, LLOQ) were tested to assess each method's accuracy and precision.

Accuracy and precision are defined as \%bias, \%CV and TE [16]. Percent bias is calculated by the percentage of an observed concentration from a nominal concentration [(measured concentration - nominal concentration)/nominal concentration*100]. Since each sample is run in multiple replicates over multiple plates, the inter- and intra-assay $\% \mathrm{CV}$ is the percent difference between the individual replicates. The sum of absolute values from these two values is the TE. To assess accuracy and precision for a pre-NME research study as in case one, only the HQC, MQC and LQC are subjected to replicates of six over three different runs.

The individual single-readout 'active' and 'total' PK methods on MSD standard gold plates showed results within the specified acceptance criteria (see Methods section for specific criteria). As shown in Figure 2, the method accuracy (\%bias) for HQC, MQC and LQC from the 'active' drug measurement is 5.0, 2.2 and $-4.0 \%$, respectively. For the 'total' drug measurement, HQC, MQC and LQC resulted in \%bias of $-4.0,3.8$ and 3.3\%, respectively. ULOQ and LLOQ results are listed in Table 1 with their respective biases and CVs. ULOQ and LLOQ biases 

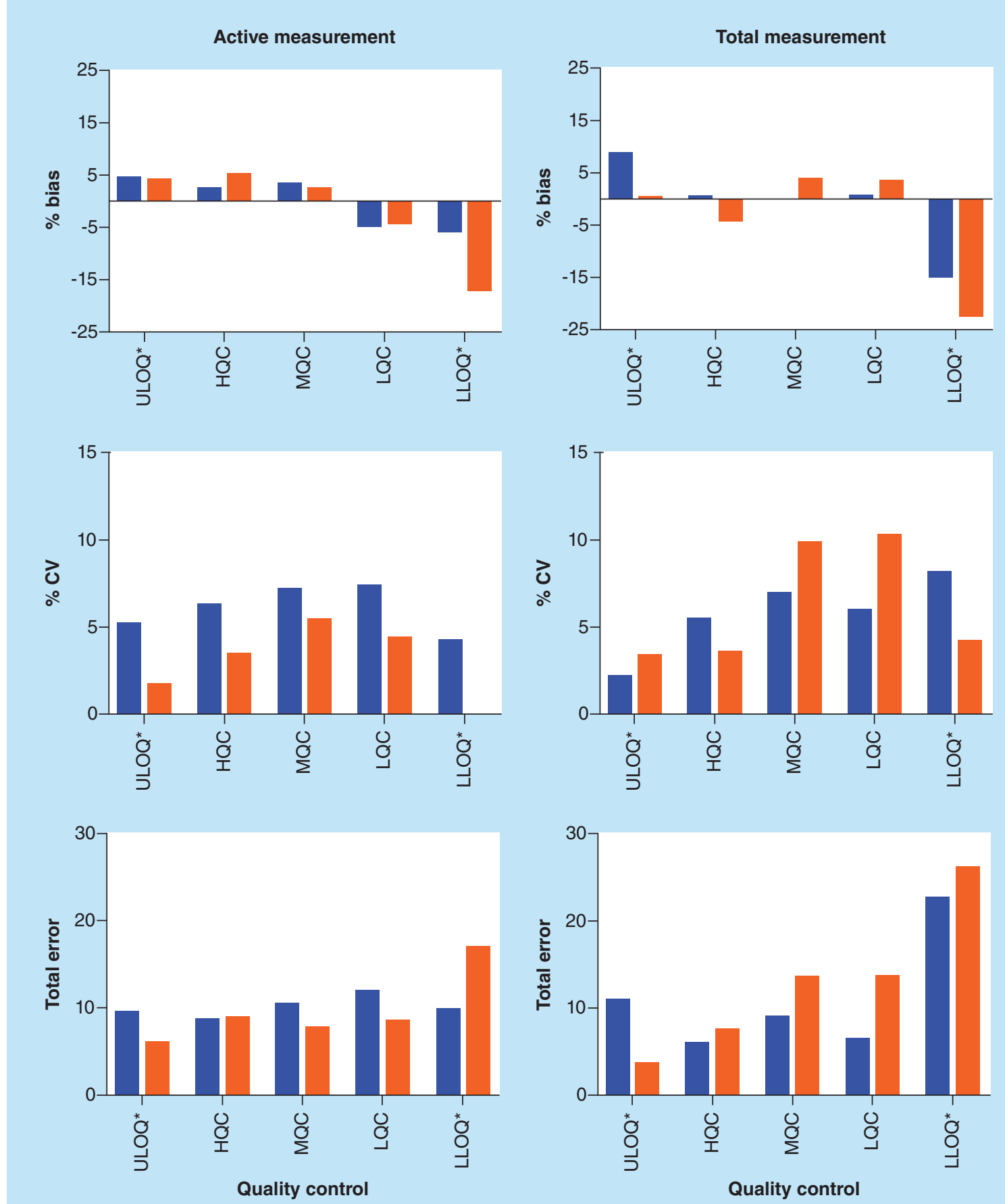

U-PLEX

Standard MSD gold

Figure 2. Accuracy and precision results for ULOQ, HQC, MQC and LQC, and LLOQ for both U-PLEX and streptavidin Meso Scale Discovery gold platforms.

*ULOQ and LLOQ only tested on replicate per research assessment acceptance guidelines.

HQC: High quality control; LLOQ: Lower limit of quantification; LQC: Low quality control; MQC: Mid quality control; ULOQ: Upper limit of quantification. 


\begin{tabular}{|c|c|c|c|c|c|c|}
\hline Dilution factor & \multicolumn{3}{|c|}{ Active results } & \multicolumn{3}{|c|}{ Total results } \\
\hline \multicolumn{7}{|c|}{ Streptavidin MSD gold results } \\
\hline $1 / 5^{\dagger}$ & $1904.59^{\dagger}$ & $0.06^{\dagger}$ & $-87.3^{\dagger}$ & $6712.01^{\dagger}$ & $0.06^{\dagger}$ & $-55.25^{\dagger}$ \\
\hline \multicolumn{7}{|l|}{ U-PLEX results } \\
\hline $1 / 5^{\dagger}$ & $4670.42^{\dagger}$ & $3.38^{\dagger}$ & $-68.86^{\dagger}$ & $7738.23^{\dagger}$ & $15.89^{\dagger}$ & $-48.41^{\dagger}$ \\
\hline $1 / 500$ & $13,091.51$ & 6.63 & -12.72 & $13,363.68$ & 0.34 & -10.91 \\
\hline $1 / 2500$ & $12,088.72$ & 4.06 & -19.41 & $12,617.50$ & 0.48 & -15.86 \\
\hline
\end{tabular}

ranged from -16.86 to $4.24 \%$ for the active measurements meanwhile -22.02 to $8.70 \%$ bias for total. ULOQ and LLOQ CVs ranged from 0.01 to $5.14 \%$ for the active measurements meanwhile $2.11-8.09 \% \mathrm{CVs}$ for total. When 'active' and 'total' drug measurement were conducted together in the multiplex assay format, HQC, MQC and LQC results also demonstrated acceptable assay performance. Percent bias ranged from -4.5 to $3.2 \%$ for 'active' drug PK method and from 0.01 to $0.4 \%$ for 'total' drug PK method. Figure 2 shows the \%bias from H/M/L QCs are comparable for both 'active' and 'total' PK methods performed separately on standard plates or in a multiplexing format.

Successful results, within acceptance criteria of regulated authorities' standards, of accuracy and precision on the U-PLEX format also indicate the drug compound and potential biotransformation's find equilibrium. This is demonstrated by yielding accurate quantification of those drug species being quantified by the 'active' also be quantified by 'total'. Further assessment of this accurate quantification can be found below in the 'Active and total confirmation' section. To complete the method assessment of this pre-NME case study, DL needs to be evaluated to confirm the integrity of a sample after multiple dilutions.

\section{Dilutional linearity}

A DL sample, prepared at $15 \mu \mathrm{g} / \mathrm{ml}$ in cynomolgus monkey plasma, represents the anticipated maximum concentration $\left(\mathrm{C}_{\max }\right)$ in study samples for this therapeutic. The $\mathrm{C}_{\max }$ sample was tested in both 'active' and 'total' PK assays performed on either standard MSD gold plates separately or U-PLEX plates together in the multiplex format. The first dilution $(1 / 5)$ is outside the range of quantification for both 'active' and 'total' methods. The second and third dilutions of $1 / 500$ and 1/2500 fall within the quantifiable range for the assays. The $C_{\max }$ sample was diluted in a 96-well deepwell plate and diluted samples were transferred to two separate gold plates as well as onto the multiplexing U-PLEX plates for 'active' and 'total' drug measurement.

As shown in Table 1, DL passes acceptance criteria for both 'active' and 'total' drug PK methods regardless of single assay or multiplexed format. This provides further confidence that both 'active' and 'total' drug PK methods can be conducted in a multiplexing format by using the same diluted samples.

As the recovery at all five QC levels and DL showed comparable results between the streptavidin MSD gold and U-PLEX platform, the U-PLEX multiplex PK methods could be brought to validation if necessary for this case study. Despite the pre-NME/research grade nature of case study one, the results would also pass the more rigorous guidelines set forth by regulatory guidelines previously mentioned. To further confirm the accuracy of the 'active' and 'total' quantifications, additional samples were created.

\section{'Active' \& 'total' measurement confirmation}

The therapeutic solution should contain 100\% 'active' drug species, so performing accuracy and precision with spiked QCs may not be enough to validate if the platform can truly distinguish and measure 'active' drug in the presence of excessive framework only drug species. To determine if the platform can quantify both drug species simultaneously, additional $\mathrm{mAb}$ framework (without the presence of the peptide) was added in varying molar ratios 
Table 2. Confirmation of 'active' and 'total' drug quantification in the presence of excess monoclonal antibody framework on U-PLEX platform.

\begin{tabular}{|c|c|c|c|c|c|c|c|c|}
\hline \multirow{2}{*}{$\begin{array}{l}\text { Molar ratio } \\
\text { (framework: } \\
\text { therapeutic) }\end{array}$} & \multicolumn{4}{|c|}{ Active results } & \multicolumn{4}{|c|}{ Total results } \\
\hline & $\begin{array}{l}\text { Nominal } \\
\text { concentration } \\
(\mathrm{ng} / \mathrm{ml})\end{array}$ & $\begin{array}{l}\text { Calculated } \\
\text { concentration } \\
(\mathrm{ng} / \mathrm{ml})\end{array}$ & $\% \mathrm{CV}$ & $\%$ Bias & $\begin{array}{l}\text { Nominal } \\
\text { concentration } \\
(\mathrm{ng} / \mathrm{ml})\end{array}$ & $\begin{array}{l}\text { Calculated } \\
\text { concentration } \\
(\mathrm{ng} / \mathrm{ml})\end{array}$ & $\% \mathrm{CV}$ & $\%$ Bias \\
\hline $10: 1$ & $11,718.75$ & $11,380.91$ & 4.93 & -2.88 & $128,906.25$ & $139,450.06$ & 1.85 & 8.18 \\
\hline $1: 1$ & $11,718.75$ & $11,534.32$ & 2.11 & -1.57 & $23,437.50$ & $23,392.21$ & 2.03 & -0.19 \\
\hline $0.1: 1$ & $11,718.75$ & $10,484.34$ & 1.85 & -10.53 & $12,890.63$ & $11,097.65$ & 1.59 & -13.91 \\
\hline $\begin{array}{l}\text { 1:0 (framework } \\
\text { only) }\end{array}$ & 0.00 & $\mathrm{~N} / \mathrm{A}$ & $\mathrm{N} / \mathrm{A}$ & $\mathrm{N} / \mathrm{A}$ & $11,718.75$ & $13,356.33$ & 3.93 & 13.97 \\
\hline $\begin{array}{l}0: 1 \text { (therapeutic } \\
Y \text { only) }\end{array}$ & $11,718.75$ & $11,895.96$ & 2.84 & 1.51 & $11,718.75$ & $11,875.38$ & 0.83 & 1.34 \\
\hline
\end{tabular}

as well as without any therapeutic to confirm the selectivity and specificity of the multiplex PK assay for measuring both 'active' and 'total' drug forms.

The ability for the multiplexing PK method to accurately measure the intended concentration for both 'active' and 'total' drug species with surrogate-mixed QCs shown in Table 2 further demonstrates the method's ability to differentiate the drug species using the different microspots. This is important given the purpose of the method is to determine the extent of biotransformation that occurs and determine the kinetics in losing the active portion of the peptide. When adding the mAb framework as surrogate 'inactive' drug form to the sample, we do see both 'active' and 'total' drug concentrations being accurately quantified (e.g., $11,718.75 \mathrm{ng} / \mathrm{ml}$ from the 'active' therapeutic is additive to the 1:1 ratio of the framework mAb yielding the 'total' drug concentration of $23,392.21 \mathrm{ng} / \mathrm{ml}$ ). The specificity of the active capture antibody to the peptide only region as well as the capture being specific to the framework only region of the therapeutic is confirmed in this experiment, allowing for monitoring of the in vivo kinetics of 'active' versus 'total' drug concentrations.

\section{Case study 2 \\ Multiplex PK method for detecting two coadministered therapeutics}

Two different mAbs, referred to as Therapeutic A and Therapeutic B, against two different cell surface receptors, commonly upregulated within the same diseased patient population, will be coadministered in a planned clinical study. The current standard of practice for developing PK methods to support clinical study requires a separate PK method to be developed and validated for each compound prior to performing bioanalysis for each coadministered compound from the study. Ensuring the combination of biologics does not cause an interference during the quantification of the therapeutics is important to measure. Having the ability to simultaneously measure two different $\mathrm{mAbs}$ in the same study sample with a single assay format can potentially improve efficiency and productivity of a bioanalytical lab via bioanalytical throughput and reducing consumables. In addition, the time to run the bioanalysis will be less and sample volume can be reduced during collection, which can be critical in oncology and pediatric studies. Drug-specific anti-idiotype antibody pairs were used to develop clinical PK methods for each compound.

\section{Accuracy \& precision}

The clinical PK methods for Therapeutic A and Therapeutic B were initially developed separately on the streptavidin MSD gold plates. Accuracy and Precision evaluation included six independent runs containing two replicates of each QC at five different concentration levels ( $\mathrm{n}=12$ per QC sample). The QC samples were prepared in $100 \%$ human serum by mixing both Therapeutic $A$ and Therapeutic B at its defined concentration as described in the 'Materials \& methods' section. QCs for each therapeutic are generally prepared as separate solutions, but to mimic a potential patient sample, the therapeutics were combined for comparison of U-PLEX to MSD gold.

The accuracy (\%bias) for Therapeutic A ranges from -2.0 to $10.6 \%$, while Therapeutic B -0.3 to $23.05 \%$ on the streptavidin MSD gold plates (Figure 3A \& D). The precision (\%CV) ranges from 4.5 to $8.5 \%$ for Therapeutic $\mathrm{A}$ and 7.7 to $9.7 \%$ for Therapeutic B in their respective method (Figure 3B \& E) and TE, the additive error between accuracy and precision, of $8.5-17.0 \%$ and $8.3-31.4 \%$, respectively (Figure $3 \mathrm{C} \& \mathrm{~F}$ ). 
(A)

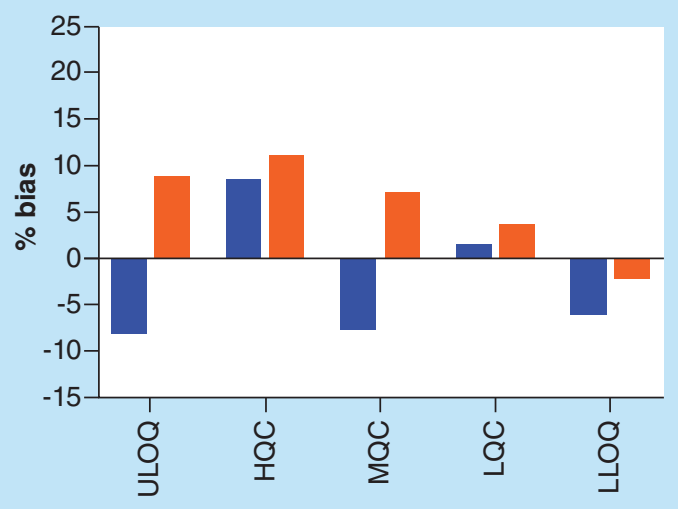

(B)

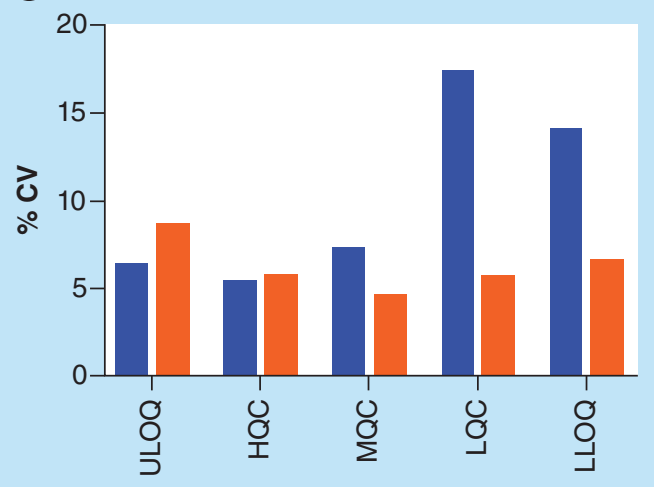

(C)

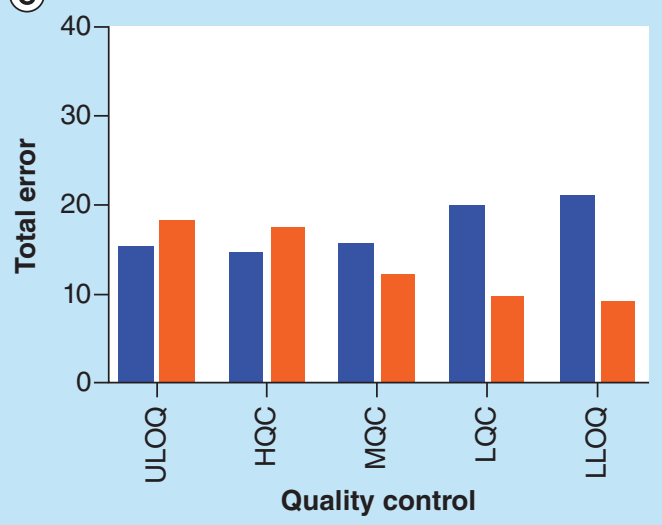

(D) Therapeutic B

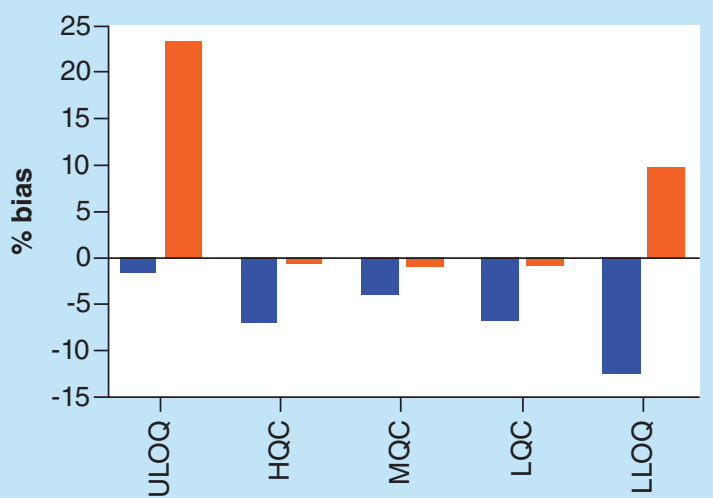

(E)

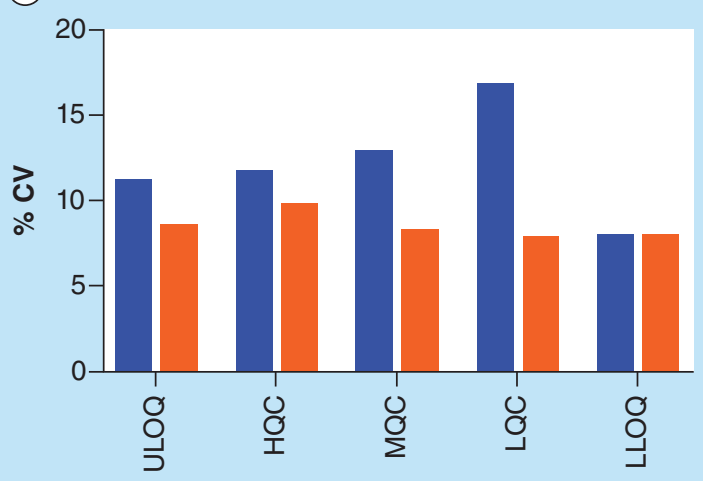

(F)

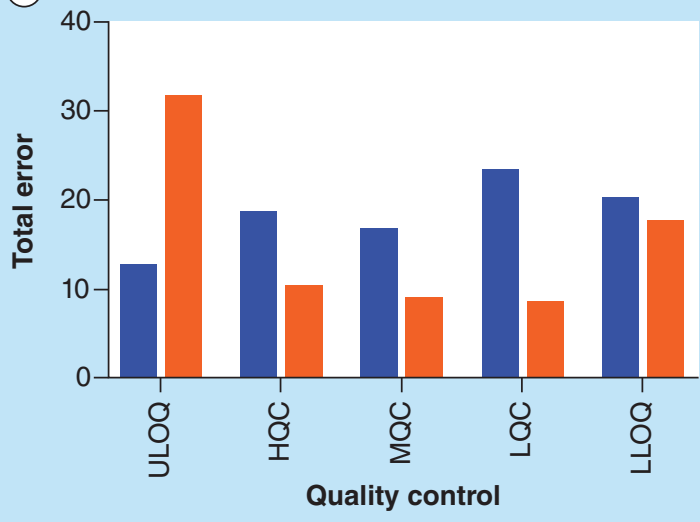

U-PLEX

Standard MSD gold

Figure 3. Results from accuracy and precision evaluation of Therapeutic A and Therapeutic B.

When multiplexing the PK methods on the U-PLEX plates, the accuracy (\%bias) for Therapeutic A ranges from -7.9 to $8.2 \%$ for all the same QCs while for Therapeutic B it ranged from -12.1 to $-1.3 \%$ (Figure $3 \mathrm{~A} \& \mathrm{D}$ ). Interassay precision $(\% \mathrm{CV})$ shows an average of 10 and $12 \%$ for Therapeutic $\mathrm{A}$ and Therapeutic $\mathrm{B}$, respectively (Figure 3B \& E). TE was 13.6-19.7\% for Therapeutic A meanwhile 8.5-17.0\% for Therapeutic B (Figure 3C \& F). 


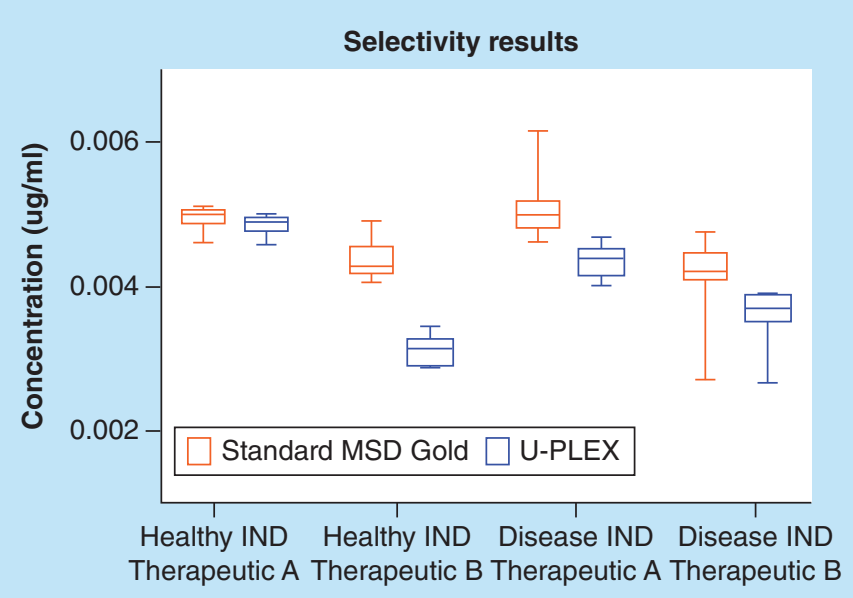

Figure 4. Healthy and disease individual's selectivity results for both therapeutics on both Meso Scale Discovery platforms.

Figure 3 shows a side-by-side analysis of the streptavidin MSD gold versus U-PLEX platform accuracy and precision performance.

The multiplex PK method for the measurement of Therapeutic A and Therapeutic B in a single sample met acceptance criteria for accuracy and precision per industry and regulatory standards.

\section{Selectivity}

Selectivity is another critical assay parameter required in method validation according to regulatory guidance. Selectivity samples were prepared in serum from ten different healthy or diseased individuals by spiking in the drug concentration at the LLOQ. A single set of selectivity sample was prepared by spiking in both Therapeutic $A$ and Therapeutic $B$ into the same sample at the lowest quantifiable concentrations for each $m A b$. The LLOQ of the PK methods for both Therapeutic A or Therapeutic B remain the same regardless of being performed on the gold plates or the U-PLEX plates. The LLOQ for Therapeutic A was $0.005 \mu \mathrm{g} / \mathrm{ml}$ while for Therapeutic B, the concentration was $0.004 \mu \mathrm{g} / \mathrm{ml}$.

Shown in Figure 4, the results of selectivity samples tested with the single-readout PK method performed on the streptavidin MSD gold plates for Therapeutic A, range from 0.0046 to $0.0051 \mu \mathrm{g} / \mathrm{ml}$ for healthy individuals and 0.0046 to $0.0062 \mu \mathrm{g} / \mathrm{ml}$ for disease individuals. Two samples from the diseased selectivity samples had results below quantifiable limit. Overall, selectivity testing on streptavidin MSD gold plates demonstrated acceptable performance for Therapeutic $A$ with $10 / 10$ and $8 / 10$ passing acceptance criteria for healthy and disease individuals, respectively. The results of selectivity samples tested on the gold plates for Therapeutic B ranged from 0.0041 to 0.0049 and 0.0027 to $0.0047 \mu \mathrm{g} / \mathrm{ml}$ for healthy and disease individuals, respectively. The selectivity testing for Therapeutic B on the gold plates was also acceptable with a passing rate of $10 / 10$ for healthy individuals and $9 / 10$ for diseased individuals.

The multiplex PK method on U-PLEX plates tested the same ten healthy individuals and ten diseased individuals now containing both Therapeutic A and Therapeutic B. Unspiked baseline serum samples from ten healthy or ten diseased subjects were also tested, with results that were below the lowest quantifiable concentration for each therapeutic. The results for selectivity samples from Therapeutic $A$ were acceptable with a passing rate of $10 / 10$ for both healthy and diseased individuals. The results for selectivity samples from Therapeutic B were also acceptable with a passing rate of $8 / 10$ of healthy individuals and $9 / 10$ of diseased individuals.

The slight difference in the passing rate between the multiplex PK method and the individual PK methods is most likely due to the assay variations such as either sample preparation, dilution, assay variability or a combination of these factors. These results demonstrated the multiplex PK method for both therapeutics maintains similar assay performance and sensitivity when compared with each single-readout MSD PK method. The importance of selectivity in clinical method development is to stress the robustness of the lowest limit of the designated range of 


\begin{tabular}{|c|c|c|c|c|c|c|}
\hline Dilution factor & \multicolumn{3}{|c|}{ Therapeutic A $(2070 \mu \mathrm{g} / \mathrm{ml})$} & \multicolumn{3}{|c|}{ Therapeutic B $(5 \mu \mathrm{g} / \mathrm{ml})$} \\
\hline \multicolumn{7}{|l|}{ U-PLEX plates } \\
\hline $1 / 40$ & $19.58^{\dagger}$ & $7.15^{\dagger}$ & $-99.05^{\dagger}$ & 4.39 & 2.29 & -12.23 \\
\hline $1 / 1600$ & 1894.84 & 2.01 & -8.46 & 4.49 & 4.36 & -10.12 \\
\hline $1 / 40^{\dagger}$ & $441.71^{\dagger}$ & $5.78^{\dagger}$ & $-78.66^{\dagger}$ & \multirow{3}{*}{\multicolumn{3}{|c|}{$\mathrm{N} / \mathrm{A}$}} \\
\hline $1 / 1600$ & 2222.75 & 4.32 & 7.38 & & & \\
\hline $1 / 64,000$ & 2169.96 & 2.35 & 4.83 & & & \\
\hline $1 / 10^{\dagger}$ & \multirow{2}{*}{\multicolumn{3}{|c|}{$\mathrm{N} / \mathrm{A}$}} & $6.66^{\dagger}$ & 4.08 & 33.21 \\
\hline $1 / 500$ & & & & 4.78 & 0.3 & -4.36 \\
\hline
\end{tabular}

quantification while in individual sources of matrix. To replicate what individuals, both healthy and diseased, tests potential study results from patients enrolled throughout Phase I to III clinical trials.

\section{Dilutional linearity}

DL was evaluated in the multiplex PK method to compare the individual PK performance for Therapeutic A and Therapeutic B. DL samples were prepared based on projected $C_{\max }$ at 2700 and $1000 \mu \mathrm{g} / \mathrm{ml}$ for Therapeutic A and Therapeutic B, respectively, when tested in their single-readout streptavidin MSD gold individual PK method. Each therapeutic had different dilution schemes to correlate with their projected concentrations and range of quantitation needed.

DL samples for the multiplex PK method were prepared by mixing Therapeutic $A$ at concentration of $2070 \mu \mathrm{g} / \mathrm{ml}$ with $5 \mu \mathrm{g} / \mathrm{ml}$ of Therapeutic B. Due to the vast difference in each therapeutics concentration, the dilution scheme was designed in a way that the last two dilutions for Therapeutic A and the first two dilutions for Therapeutic B will be within the quantifiable range of each standard curve. In the multiplex method, only one dilution scheme can be applied for the combination study samples further complementing the efficiency of the multiplexing PK method. The DL samples had acceptable recovery for both Therapeutic A and Therapeutic B when tested in the single-multiplexed PK method as shown in Table 3 for the dilutions within the assay quantitation ranges.

Since clinical method development and validation occurs prior to protocol finalization, no study samples were available for incurred sample repeat (ISR) sample testing. In lieu of study samples, the DL sample created for the U-PLEX testing was tested in the respective previously validated streptavidin MSD gold plate methods. Table 3 shows the difference of the DL sample being quantified on the U-PLEX platform versus separately with their previously validated MSD gold platform. Therapeutic A maintained the same serial dilution scheme, meanwhile Therapeutic B was a 10/50/10 scheme. Even with the dilution scheme difference acknowledged, Table 3 shows samples passing acceptance criteria. DL tests the integrity of a sample even after being diluted multiple times. Timepoints from clinical trials at $\mathrm{C}_{\max }$ concentration often need multiple dilutions to fall on the standard curve's range of quantification.

\section{One-week linker stability testing}

Reagent stability plays a critical role in the robustness and reproducibility of PK method performance. Additionally, robust reagent stability can provide convenience and flexibility when executing bioanalysis testing.

Since multiplex PK method uses a microspot-specific linker, it is a critical reagent for positioning the capture antibody onto its designated placement in each well. The solution with the specific spot linker coupled with the desired capture reagent is further noted as the 'coupled antibody solution'. The preparation of this coupled antibody solution can be found in the 'Materials \& methods' section [8]. The storage stability of the coupled antibody solution was tested by storing at $2-8^{\circ} \mathrm{C}$ for 1 week. To evaluate the coupled antibody solution stability, standard calibrators were prepared on the same day as the coupled antibody solution was prepared and tested as the week 0 baseline 
(A)

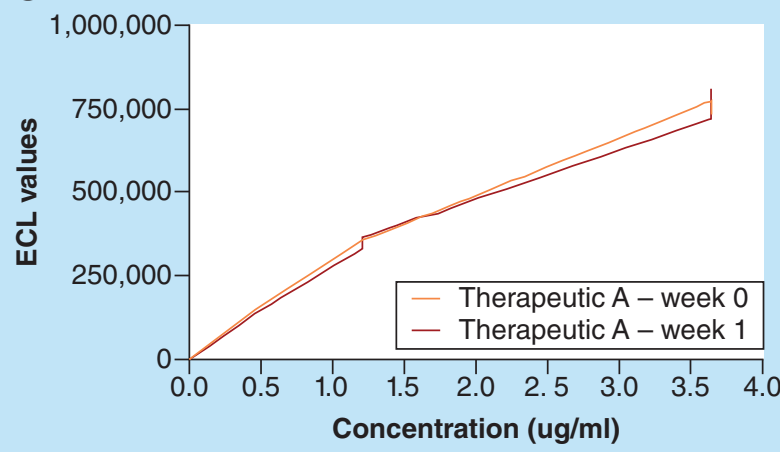

(C)

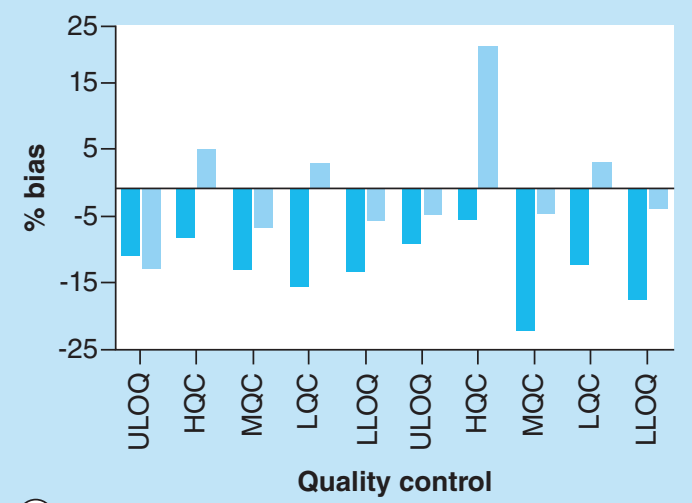

(D)

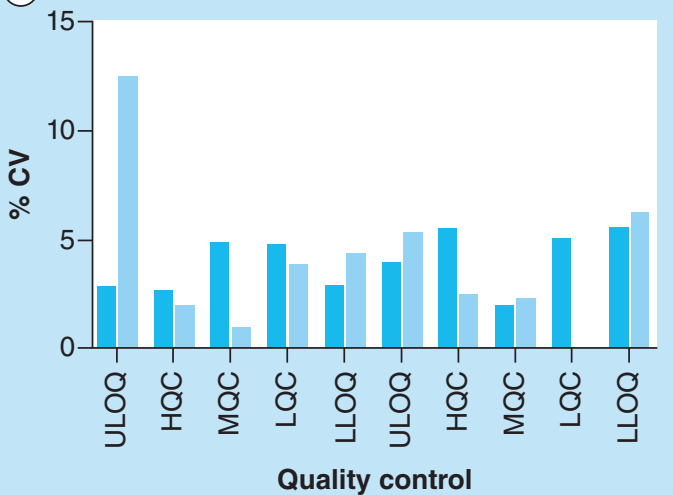

(B)

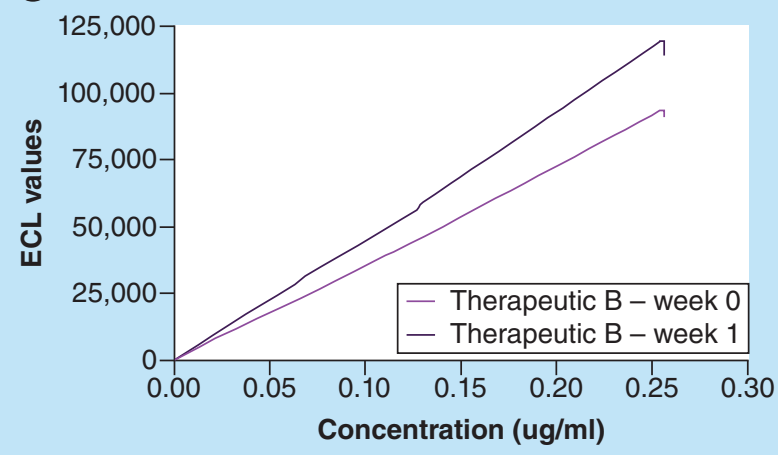

(E)

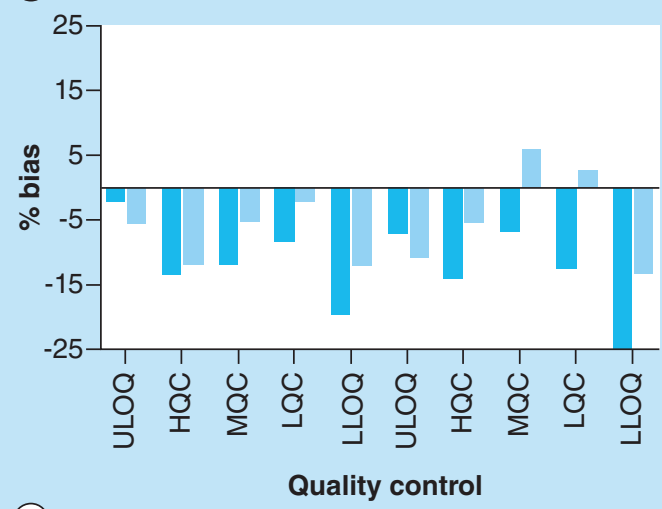

(F)

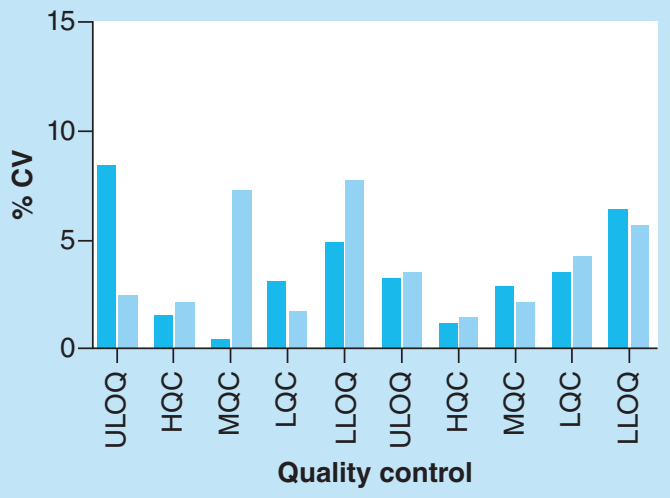

Week 0

Week 1

Figure 5. 1-week linker stability testing results. ECL values for week 0 and week 1 (A \& B) with respective quality control recoveries from week 0 and week 1 (C-F) of linker stability testing.

$\mathrm{ECL}$ : Electrochemiluminescence.

results. The coupled antibody solutions for Therapeutic $A$ and Therapeutic $\mathrm{B}$ were returned for storage at $2-8^{\circ} \mathrm{C}$ for up to week after initial run as week 0 . After 1-week storage, the coupled antibody solutions were tested in the multiplex PK method again with a fresh set of standard calibrators prepared the day of testing week 1 . As shown in Figure 5, there is no change in standard calibrator signal when comparing the week 0 versus week 1. 
The stored coupled antibody solution showed little to no difference in raw ECL signal for each therapeutic standard curve, seen in Figure 5, which also leads to little difference in QC recovery between the 2 days indicating the stored solution can be used in bioanalytical testing over this timeframe. Allowing the pre-prepared solution to be stored at $2-8^{\circ} \mathrm{C}$ can provide flexibility in performing the method and efficiency in batch size testing during bioanalysis.

\section{Discussion}

A trend toward increased development of complex biological molecules as well as the popularity of combination therapies represents an ongoing challenge to bioanalytical laboratories. Bioanalytical labs are tasked with providing sensitive and robust methods in the face of accelerated turnaround demands without sacrificing high-quality study data $[17,18]$. During the discovery stage for lead candidate selection of complex modalities, quite often multiple drug forms (intact and associated degraded forms) will have to be monitored during in vivo animal studies to establish a relationship between PK/PD, toxicity and biotransformation [19,20]. At this time, there are more than 70 Fc-fusion therapeutics approved in major markets and an additional 40+ in preclinical development [21]. Monitoring for varying drug forms, both intended drug product and its degraded drug forms, in preclinical development would ensure the best lead candidate is chosen to move into clinical development. Furthermore, it can provide critical data toward the most reliable dose prediction and PK/PD relationships for first-in-human studies. Extending the platform in preclinical development to be applied to therapeutic antibody development to assess free and total PK measurements for therapeutics with a soluble target component as well as determination of active multitarget binding in vivo for bispecific and multispecific antibody molecules would expand the platforms usability. Traditionally, PK methods tested for case studies 1 and 2 would undergo development on a single-readout platform (i.e., streptavidin MSD gold) to measure a single drug form. If the multiplexed PK method could demonstrate free and total PK along with binding or pharmacodynamic data, this would dramatically increase the understanding of the lead candidate at an earlier timepoint in development. The limiting factor for this platform's use in a preclinical animal models would be having the correct and comparable soluble targets. However, by the time the lead candidate therapeutic is in clinic, the multiplexed platform could include each of these testing types (free PK, total PK, soluble target). Using this platform for multiplexed testing also aids in the volume of sample needed to collect. Research samples from small animal or nonhuman primate studies often have low volume and may be subjected to multiple methods to quantify each drug form; this process is inefficient and may be additionally hindered by the stability of the molecule. There are defined blood collection restrictions that impact total sample volume for both nonclinical sample and targeted clinical populations (e.g., oncology and pediatric patients) in which additional aliquots or adequate sample volume may not be available. Having the ability to quantify multiple readouts by a single sample dilution is also the most efficient for bioanalytical labs, as well as can eliminate additional sample variability by being tested on separate plates at separate times.

The successful feasibility development of multiplex PK methods using the U-PLEX platform combines the known reproducibility and sensitivity from MSD technology with a substantial improvement in both speed of method development and bioanalysis throughput. Method development of a PK LBA depends heavily on dosing regimen as well as the structure of the therapeutic molecule. Since the multiplex PK methods described have comparable assay performance regarding critical assay parameters (e.g., accuracy, precision, DL and selectivity) to the individual methods developed on the standard MSD gold plates, these methods are interchangeable and will provide comparable data on drug concentrations, for example, the comparison in Table 3.

In the first case study presented focused on measuring different drug forms. The use of multiplexing LBAs provided insight into the in vivo biotransformation resulting in varying drug species within a study sample. Often the molecular variation between potential lead candidates can be small, such as a single amino acid substitution or glycosylation profile, yet the change may have significant impact on their biological effect in vivo due to a change in binding affinity or epitope specificity [15]. In addition, given complex molecule structures (e.g., bi- and multispecific biologics), a compound can have more than one 'active' moiety [22] or region responsible for biological function. To better define the structure-activity relationship, several ligand-binding formats targeting distinct regions of the molecule can help characterize if and for how long the active moiety is present in vivo [23]. From the analytical perspective in case study 1, the same standard calibrators and QC concentration levels/dilutions could be used to compare the assay performance between the multiplex PK methods to the individual MSD PK methods. Using the same standard calibrators and QCs avoids issues related to assay variation due to different standard and QC dilution schemes and allows for a direct comparison of the same diluted samples tested for each method under 
evaluation. Critical assay parameters tested, (i.e., accuracy, precision, DL), were comparable between the multiplex PK method using U-PLEX plates and the two individual PK methods using gold plates for 'active' and 'total' drug measurement. With the assay performance demonstrated by these critical assay parameters, it is likely that the multiplex PK method could be further validated according to current regulatory guidance and be implemented for bioanalysis to support any regulated studies planned. The multiplex PK method presented improved efficiency and provided greater comparison due to the diluted study samples being tested once to quantify both 'active' and 'total' drug concentration from a single sample. This type of information on the drug product is traditionally obtained by performing two independent sample analyses according to the individual PK methods needed to gather the data.

Similarly, in the second case study aimed at developing a single PK method to support clinical study analysis testing combination therapy of two coadministered biologics, a multiplexed PK method demonstrated its advantage of improving throughput in bioanalysis. The number of standard MSD gold plates used was double that of the U-PLEX plates when the DL sample was run in a side-by-side comparison; to run the MSD gold procedure additional DL volume, automation time and personnel resource time were necessary to complete the experiments. Since both therapeutics could not be completed on a single plate in a single-readout platform, like the streptavidin MSD gold, it equates to double the amount of time needed to complete in comparison to the U-PLEX experiment. To extrapolate this impact to a potential clinical study setting, the use of multiplexed methods could reduce the equivalent study analysis time in half for studies, which could include thousands of sampling time points to be tested. The results from testing the critical assay parameters (i.e., accuracy, precision, DL and selectivity) were comparable between the multiplex U-PLEX PK method designed for measurement of both therapeutics and the two previously validated individual PK methods for each therapeutic.

One of the biggest design challenges in developing multiplex PK methods is anticipating drug concentrations based on dosing regimens and schemes, which can result in different ranges of quantification. For instances, like case study 1 , this is not a design nor development challenge. In the instance of case study 2 , this can be a large challenge. Development for coadministered biologics in a single sample requires anticipating potential vast differences in nominal concentrations between the biologics (e.g., one biologic being dosed much higher or more frequently than the other resulting in a significantly higher $\mathrm{C}_{\max }$ ). Testing nominal concentrations of the biologics spiked at the anticipated individual exposure ranges can confirm the presence or nonpresence of any potential therapeutic-therapeutic assay interference. Lastly, the inefficiency of single PK readouts for coadministered study bioanalysis also allows for additional variability given that different dilution schemes can lead to sample dilutions being completed independently per therapeutic quantification.

While multiplexing PK methods can be easily adapted for discovery or research use, the development and implementation of multiplexing for more regulated studies can present additional bioanalytical challenges and capabilities. First, the programming of automated liquid handlers to create two-therapeutic standard calibrators and QCs would be necessary to eliminate additional points of potential error. Second, the determination of method for samples that only contain a single therapeutic would need to be defined. In instances where therapeutics have standalone streptavidin MSD gold procedures implemented prior to coadministration studies, additional parameters in the bioanalytical plan might need to include both multiplexed and single-readout testing. The need for both assays would be unnecessary if the U-PLEX results of single compounds also passed acceptance criteria. Since the combination standard curve, QCs and DL were all used during case study 2, we can determine that even if a sample was mixed, the standalone readout as well as the U-PLEX are both acceptable means of therapeutic quantification.

To assess the U-PLEX assay performance for use of coadministered studies, the DL samples for the multiplex PK method were spiked at the anticipated $\mathrm{C}_{\max }$ and trough concentrations for both Therapeutic $A$ and Therapeutic $B$, respectively. A common dilution scheme was designed to capture Therapeutic $\mathrm{B}$ concentrations with the first two dilutions and Therapeutic $\mathrm{A}$ concentrations with higher dilutions, corresponding with the much higher planned dose of the compound. This $\mathrm{C}_{\max }$ versus trough sample assessment was designed to mimic in vivo samples given the anticipated study design around dose concentration and regimen. Due to the added efficiency of the multiplex U-PLEX method, all study samples can be diluted at all four dilutions and tested once to measure all expected drug concentrations of both Therapeutic A and Therapeutic B.

While DL is a critical assay parameter, so is parallelism and specificity to further demonstrate sample integrity and selective reagent binding. Parallelism defined by the FDA is testing which 'demonstrates that the serially diluted incurred sample response curve is parallel to the calibration curve. Parallelism is a performance characteristic that can detect potential matrix effects and interactions between critical reagents in an assay' [6] In short, this means the 
ability for a sample to undergo different dilution schemes without showing interference of the sample outcome. Since there are no samples for this study at the time of validation completion, the use of the DL with varying dilution schemes covers the concept of parallelism for the clinical study. The DL sample testing for case study 2 addressed these additional performance attributes of clinical validation testing. First, the modified sample dilution scheme for Therapeutic B relative to the validated scheme for Therapeutic A allows clinical samples to undergo a single sample dilution scheme in the multiplexed PK method. For example, if the final sample dilution is $1 / 500$, parallelism would be demonstrated by diluting a sample by completing two dilutions of $1 / 10$ and $1 / 50$, as well as two dilutions of $1 / 25$ and $1 / 20$. Changing the dilution scheme for Therapeutic $B$ from $1 / 10,1 / 50$ and a final $1 / 10$ in the validated standalone PK method to three sequential $1 / 40$ dilutions in the U-PLEX method demonstrates Therapeutic B can undergo these dilution scheme changes without change to the data integrity. Second, cross-therapeutic interference was a concern for samples with respective high and low concentrations of each therapeutic and to test this, the multiplexed DL sample was created. The sample mimics when Therapeutic A is at its $C_{\max }$ and Therapeutic $B$ is $10 \times$, it is anticipated trough concentration. When high serum concentrations of Therapeutic A are anticipated in samples, we expect the detection of Therapeutic B at low concentration to be unaffected. At no point during the proposed study design would Therapeutic B have equal or higher concentration than Therapeutic A. The results of the DL experiment show there is no interference on the detection of Therapeutic $B$ in this instance. Based on the proposed dosing details of the planned coadministration study and the known half-life of the two compounds, there would be no instance where the serum concentration of Therapeutic A would be expected to be lower than Therapeutic B. Thus, providing justification for testing the molar ratio described in the DL sample as the only necessary control for specificity in the multiplex PK method.

Validated assays also assess other additional criteria to demonstrate robustness; these criteria were evaluated for the coadministration assay development method. Specificity, selectivity and stability are important robustness features of validated assays. Specificity determines if there are any other binding agents in the sample, which can interfere with the QC recovery. Selectivity proves the lowest point of quantification can be recovered from ten individual samples. Stability of the diluted samples or reagents can provide ease of clinical analysis during bioanalytical batch testing. Specificity between the coadministrated therapeutics A and B, as previously discussed when evaluating DL sample recovery, can also be assessed when evaluating the QC recoveries. A separate preparation of specificity was not necessary since the QCs cover a range of mixed concentrations. For example, where Therapeutic A's ULOQ is $145.8 \mu \mathrm{g} / \mathrm{ml}$, Therapeutic B is approximately $10.24 \mu \mathrm{g} / \mathrm{ml}$. Since the anticipated dosing regimen is always much higher for Therapeutic A versus Therapeutic B, the need to test where Therapeutic B is high and Therapeutic A is low would not be necessary since it is unlikely this situation would occur in clinical samples. However, where samples might be similar, we can look at the LLOQ QC. In the LLOQ sample, Therapeutic A is $0.2 \mu \mathrm{g} / \mathrm{ml}$ and Therapeutic B is $0.16 \mu \mathrm{g} / \mathrm{ml}$. Selectivity is completed in a typical LBA validation to prove that at the LLOQ concentration, individuals as will be tested during the study can recover in comparison to the pooled matrix. Selectivity tested in ten healthy and ten diseased individuals were compared with previously validated results, although new ones were prepared for U-PLEX testing. The new set was unable to be tested on the streptavidin gold plates for direct comparison due to the change in MRD to Therapeutic B. Due to this, a direct correlation is difficult to draw between previously validated results of single-readout PK to U-PLEX testing results. However, since both sets of selectivity samples across both platforms pass acceptance criteria, we can conclude comparability of the assay performance at these lower levels. Stability testing in validated methods is important to complete to better understand the stability of the compound, the study sample and the collected matrix. Understanding these profiles give a window of when the bioanalysis must be completed, and how it will be completed. QCs at different stressed states (e.g., varying temperatures, freeze-thaw cycles, stored diluted samples) define the bioanalysis parameters in which study samples will then follow. A stressed stability can also be applied to master mix solutions, so in the case of the U-PLEX platform, the coupled-antibody solution was used for reagent stability. The results from the 1-week stability testing of the coupled-antibody solution demonstrate this reagent to be as effective as fresh prepared solution even after stored for 1 -week at $4{ }^{\circ} \mathrm{C}$ when comparing ECL values of the standard curve and QC recoveries. This stability test helps mitigate another point of potential variation as well as amplifies time saving during bioanalysis. The possibility of creating one large batch of coupled-antibody solution can aid in clinical bioanalytical use. The coupled-antibody solution takes approximately $1 \mathrm{~h}$ to create, so creating it in a large batch can streamline the bioanalytical process.

During development for both multiplexing methods, case study 1 for lead candidate optimization and case study 2 of the cospiked therapeutics, the potential difference in plate chemistry between streptavidin MSD gold and 
U-PLEX was considered. The U-PLEX plates, whose plates are the same in overall shape as a streptavidin MSD gold plate, is comprised of ten unique spots within a single well. These ten spots have a much smaller surface area for binding. A streptavidin MSD gold plate has $0.3 \mathrm{pmol} /$ well binding capacity meanwhile the same size well has $0.1 \mathrm{pmol} /$ well binding capacity for the U-PLEX platform. In addition to the reduced size, the spot needs to bind to a specialized linker prior to biotin capture occurring. This chemistry and binding capacity change the dynamic of the complex in comparison to a full streptavidin spot. This could also change the amount of sample that is added to the well prior to saturation occurring. While keeping this in mind, we focused on maintaining the concentrations of the biotin-labeled antibodies from the previously validated MSD gold plates in order to have a direct comparison between the two platforms. We noted that the U-PLEX platform could have been pushed to a lower range of quantification for these case studies based on signal-to-noise separation at low calibrator concentrations, however, assay development also needed to be attainable on streptavidin MSD plates for comparative purposes. From this, we can suspect the coupled-antibody solution is highly saturated, thus ensuring the linker spot is also completely occupied with the intended capture antibody. After optimizing the reagent needs based on these plate differences, overall sample volumes should also be considered during sample preparation when comparing the two platforms. To run multiple methods with streptavidin MSD gold plates, the samples would need to be plated twice resulting in a twofold increase in sample volume necessary for plating. Although adult clinical samples generally have these types of volumes available, nonclinical studies or pediatric sample volumes are much lower volumes. The U-PLEX platform can be beneficial in this instance. The importance of being able to discern two separate characteristics in a single sample is increasingly necessary with complicated structured therapeutics. In addition, being able to define if a therapeutic has a potential point of degradation, whether it is due to clipping, oxidation or other in vivo causation, it is important to understand under at what timepoint this occurs. Determining active and total from a single sample demonstrates that your therapeutic is following the anticipated half-life, however, your therapeutic might also no longer be binding to its intended target. In addition, determining if one therapeutic is interfering the quantification of other coadministered therapeutic in the study sample can be determined by their baseline half-lives using the U-PLEX methods. To further the use of the U-PLEX platform, additional capabilities could be test biomarkers at the same time as PK if additional testing proved no interference would occur.

\section{Conclusion}

Having demonstrated comparable assay performance of multiple methods through the testing of the described acceptance criteria on the U-PLEX platform opens the door to increasing the efficiency of current bioanalytical laboratory practices. The implementation of the U-PLEX platform in early development has already been shown to reduce anti-idiotype reagent screening time by tenfold in the same working time as a streptavidin MSD gold plate [24]. Even using a 2-spot assay, utilizing only two of the ten unique spots on the U-PLEX plate, allows multiplexing PK methods for drug form testing and coadministration studies reinforces the efficiency, quality and cost drivers for this bioanalytical strategy [18]. When doing a cost analysis of the U-PLEX Development Pack 2-Assay (Catalog: K15227N-4) versus streptavidin gold SECTOR 96-well Plates (Catalog: L15SA-7), we see little difference when breaking down to a per therapeutic cost. The raw materials cost difference between streptavidin gold SECTOR plates and the U-PLEX Development Pack 2-Assay breaks down to approximately a $\$ 20$ increase when using the U-PLEX platform. However, factoring in the use of one analyst versus two, and the time savings to complete the multiplexed bioanalysis, the U-PLEX saves a significant amount of operating costs. The reduction in consumables, and more importantly, personnel or analyst's time more than compensates for the increased plate cost between the streptavidin MSD gold plates and the U-PLEX plates.

Overall, the U-PLEX platform can be considered for a fit-for-purpose LBA option for nonclinical and clinical bioanalysis for drug forms and coadministered use. The U-PLEX after close analysis to the standard MSD gold would be more beneficial for high-throughput bioanalysis in a cost-efficient manner. The implementation in research use only studies can begin immediately meanwhile the use in qualified and validated assays will need additional uniformity to meet current industry guidelines.

\section{Future perspective}

Implementation of multiplex PK immunoassay can be critical in the reduction of resource needs, both consumable waste as well as human resourcing. The high demand for additional information regarding complex molecules and coadministered studies can be completed using U-PLEX 2-spot plates yielding robust results in half the time. Current robotic platforms will need additional programing to handle the two-drug assays, meanwhile are already 
equipped for the detection of drug degradants via biotransformation drug development. This novel platform can aid in the sensitivity and robustness of assays when in comparison to previously used LC-MS assays in detecting multiple drug degradants or drug species, as well as overall throughput.

\section{Summary points}

- Multiplex pharmacokinetic immunoassays validated by regulatory authority standards for fit-for-purpose nonclinical animal study for quantification of drug degradants caused by in vivo biotransformation.

- 'Active' and 'total' drugs noted to represent a quantification of drug species with active binding to the intended target while also quantifying the total Fc in circulation.

- Since the biotransformation can lead to a decrease of active species, specificity-like testing with additional framework completed and demonstrated the U-PLEX ${ }^{\circledR}$ able to differentiate the concentrations in these samples.

- Multiplex pharmacokinetic immunoassays validated by regulatory authority standards for clinical coadministration studies.

- Case study to demonstrate bioanalysis of coadministered therapeutics demonstrated accuracy and precision, dilutional linearity, selectivity and specificity.

- Stability of unique U-PLEX solution also demonstrated in clinical case study to prove ease of bioanalysis for future efforts.

Financial \& competing interests disclosure

The authors have no relevant affiliations or financial involvement with any organization or entity with a financial interest in or financial conflict with the subject matter or materials discussed in the manuscript. This includes employment, consultancies, honoraria, stock ownership or options, expert testimony, grants or patents received or pending, or royalties.

No writing assistance was utilized in the production of this manuscript.

\section{References}

Papers of special note have been highlighted as: $\bullet$ of interest; $\bullet \bullet$ of considerable interest

1. Lachno DR, Emerson JK, Vanderstichele $\mathrm{H}$ et al. Validation of a multiplex assay for simultaneous quantification of amyloid-beta peptide species in human plasma with utility for measurements in studies of Alzheimer's disease therapeutics. J. Alzheimers Dis. 32(4), 905-918 (2012).

2. Cuyckens F. Mass spectrometry in drug metabolism and pharmacokinetics: current trends and future perspectives. Rapid Commun. Mass Spectrom. doi:10.1002/rcm.8235 (2018) (Epub ahead of print).

3. Wijnant GJ, Van Bocxlaer K, Fortes Francisco A et al. Local skin inflammation in cutaneous leishmaniasis as a source of variable pharmacokinetics and therapeutic efficacy of liposomal amphotericin B. Antimicrob. Agents Chemother. 62(10), pii:e00631-18 (2018).

4. Chowdhury F, Williams A, Johnson P. Validation and comparison of two multiplex technologies, Luminex and Mesoscale Discovery, for human cytokine profiling. J. Immunol. Methods 340(1), 55-64 (2009).

5. Krohne TU, Muether PS, Stratmann NK et al. Influence of ocular volume and lens status on pharmacokinetics and duration of action of intravitreal vascular endothelial growth factor inhibitors. Retina 35(1), 69-74 (2015).

6. Department of Health and Human Services, United States. Food and Drug Administration, Center for Drug Evaluation and Research (U.S.), \& Center for Veterinary Medicine (U.S.). Bioanalytical Method Validation Guidance for Industry. (2018). www.fda.gov/media/70858/download

- Guidelines of the US FDA for bioanalytical method validation is of interest as the acceptance criteria for these case studies are based on these guidelines.

7. Lagasse HA, Alexaki A, Simhadri VL et al. Recent advances in (therapeutic protein) drug development. F1000Res 6, 113 (2017).

8. Meso Scale Diagnostics LLC. U-PLEX assay platform (2018). www.mesoscale.com/en/products_and_services/assay_kits/u-plex_gateway/u-plex_technology

9. Tang H, Shah K, Steinmetz T et al. Development and validation of a multiplexed drug level assay in support of combination biologics therapy clinical studies. J. Pharm. Biomed. Anal. 171, 204-211 (2019).

-• It also supports the use of case study 2 . It is additionally able to measure incurred samples during testing.

10. Sugahara KN, Teesalu T, Karmali PP et al. Coadministration of a tumor-penetrating peptide enhances the efficacy of cancer drugs. Science 328(5981), 1031-1035 (2010).

11. Piccolo MT, Menale C, Crispi S. Combined anticancer therapies: an overview of the latest applications. Anticancer Agents Med. Chem. 15(4), 408-422 (2015).

- Guidelines of the FDA for bioanalytical method validation is of interest as the acceptance criteria for these case studies are based on these guidelines. 
12. Iyoda T, Nagamine Y, Nakane Y et al. Coadministration of the FNIII14 peptide synergistically augments the anti-cancer activity of chemotherapeutic drugs by activating pro-apoptotic bim. PLoS ONE 11(9), e016252 (2016).

13. Messenheimer DJ, Jensen SM, Afentoulis ME et al. Timing of PD-1 blockade is critical to effective combination immunotherapy with anti-OX40. Clin. Cancer Res. 23(20), 6165-6177 (2017).

14. Westin JR, Chu F, Zhang M et al. Safety and activity of PD1 blockade by pidilizumab in combination with rituximab in patients with relapsed follicular lymphoma: a single group, open-label, Phase II trial. Lancet Oncol. 15(1), 69-77 (2014).

15. Laurenzana EM, Stevens MW, Frank JC et al. Pharmacological effects of two anti-methamphetamine monoclonal antibodies. Supporting data for lead candidate selection for clinical development. Hum. Vaccin. Immunother. 10(9), 2638-2647 (2014).

16. DeSilva B, Smith W, Weiner R et al. Recommendations for the bioanalytical method validation of ligand-binding assays to support pharmacokinetic assessments of macromolecules. Pharm. Res. 20(11), 1885-1900 (2003).

17. Watson RG, Clements-Egan A, Schantz A et al. Implementing a tiered approach to bioanalytical method validation for large-molecule ligand-binding assay methods in pharmacokinetic assessments. Bioanalysis 9(18), 1407-1422 (2017).

18. Unger S, Lloyd T, Tan M, Hou J, Wells E. The science of laboratory and project management in regulated bioanalysis. Bioanalysis 6(10), 1357-1372 (2014).

19. Zhang J, Hong Y, Shen J. Combination treatment with perifosine and MEK-162 demonstrates synergism against lung cancer cells in vitro and in vivo. Tumour Biol. 36(7), 5699-5706 (2015).

20. Thomas RS, Philbert MA, Auerbach SS et al. Incorporating new technologies into toxicity testing and risk assessment: moving from 21 st century vision to a data-driven framework. Toxicol. Sci. 136(1), 4-18 (2013).

21. Strohl WR. Current progress in innovative engineered antibodies. Protein Cell 9(1), 86-120 (2018).

22. Chaubal MV. Application of drug delivery technologies in lead candidate selection and optimization. Drug Discov. Today 9(14), 603-609 (2004).

23. Neitz RJ, Chen S, Supek F et al. Lead identification to clinical candidate selection: drugs for Chagas disease. J. Biomol. Screen. 20(1), 101-111 (2015).

24. Ware M, Shankar G, Breslin P, Yang TY. Application of automated liquid handling in ligand-binding assay-based bioanalytical method development: the practical considerations. Bioanalysis 10(7), 425-430 (2018). 